\title{
Anti-Inflammatory Effect of Feiyangchangweiyan Capsule on Rat Pelvic Inflammatory Disease through JNK/NF- $\kappa$ B Pathway
}

\author{
Yao Li, ${ }^{1}$ Yang Liu, ${ }^{2}$ Qian Yang, ${ }^{3}$ Zhihui Shi, ${ }^{4}$ Yanhua Xie $\mathbb{D},{ }^{3}$ and Siwang Wang $\mathbb{D}^{3}$ \\ ${ }^{1}$ School of Pharmacy, Shaanxi University of Chinese Medicine, Xianyang 712000, China \\ ${ }^{2}$ College of Chemistry and Pharmacy, Northwest Agriculture and Forestry University, Yangling 712100, China \\ ${ }^{3}$ Department of Natural Medicine, School of Pharmacy, The Fourth Military Medical University, Xian 710032, China \\ ${ }^{4}$ Shaanxi Junbisha Pharmaceutical Limited Company, Shaanxi, China
}

Correspondence should be addressed to Yanhua Xie; xieyanh@fmmu.edu.cn and Siwang Wang; wangsiw@fmmu.edu.cn

Received 8 November 2017; Revised 22 January 2018; Accepted 24 January 2018; Published 28 February 2018

Academic Editor: Kuzhuvelil B. Harikumar

Copyright (c) 2018 Yao Li et al. This is an open access article distributed under the Creative Commons Attribution License, which permits unrestricted use, distribution, and reproduction in any medium, provided the original work is properly cited.

\begin{abstract}
Objectives. In this study, we aimed to illustrate the preventive effect and possible mechanisms of Feiyangchangweiyan capsule (FYCWYC) on rat pelvic inflammatory disease (PID) model. Methods. To construct the rat PID model, upper genital tract was infected by multipathogen, and then drugs were orally administered for 8 days. The histological examination, immunohistochemical analysis, and ELISA were carried out. Furthermore, Western blotting was used to analyze the expression of Akt, MAPKs, NF- $\kappa \mathrm{B}$ p65, and $\mathrm{I} \kappa \mathrm{B}-\alpha$ in uterus. Results. As the results showed, infiltrations of neutrophils and lymphocytes in uterus were significantly suppressed, and IL-1 $\beta$, IL-6, CXCL-1, and TNF- $\alpha$ were also reduced in a dose-dependent manner. We also found that FYCWYC inhibited apoptosis induced by infection. Furthermore, FYCWYC could block the infection-induced nuclear translocation of NF$\kappa \mathrm{B}$. We found that FYCWYC treatment only decreased the phosphorylation of JNK induced by infection and had no effects on Akt and P38. Additional, the effects of SP600125, an inhibitor of phospho-JNK, were similar to the results of FYCWYC. Conclusions. Taken together, our results demonstrated that FYCWYC had anti-inflammatory effect in pathogen-induced PID model, and the mechanism might be through inhibiting NF- $\kappa$ B nuclear translocation which is mediated by JNK.
\end{abstract}

\section{Introduction}

Chronic pelvic inflammatory disease (PID), which is caused by ascending pathogenic microorganisms infection in the upper female genital tract, is a commonly observed distress in female genital system [1]. PID can lead to tubal factor infertility, ectopic pregnancy, chronic pelvic pain, and some others serious sequelae, and it has been considered a great threat to the women's life quality. Its symptoms include salpingitis, endometritis, and peritonitis [2]. The results from hematoxylin and eosin-stained experiment showed that there are many neutrophils and lymphocytes infiltrations in pathogenic sites, and this could be used as a criterion in diagnosing PID in clinic [3]. The common pathogens of PID include Gram-negative bacteria, mycoplasmas, trachomatis, Gram-positive bacteria, and Neisseria gonorrhoeae [4-6]. To treat PID, antibiotic regimens are often used in clinical practice, but antibiotics often induce antibiotic resistance [7].
Therefore, it is necessary to develop new complementary and alternative medicine for PID treatment.

Feiyangchangweiyan capsule (FYCWYC), a popular Chinese medicinal formula, is composed of Euphorbia hirta L., Polygonum chinense L., and Ilex rotunda Thunb. In clinical settings, FYCWYC has been used in removing toxin, and treating bacteria induced bacillary dysentery, acute, and chronic gastroenteritis [8]. During patient follow-up, we found that many PIDs were alleviated, even cured. This information was very interesting, so we want to know the reasons.

Most of constituents of medicinal plants in FYCWYC have potential antimicrobial and anti-inflammatory activities. Euphorbia hirta L. exerts some pharmacological effects, including antipyretic, analgesic, and anti-inflammatory properties in rat and mice model [9]. The methanolic and ethanolic extracts of Euphorbia hirta L. also have antifungal and antibacterial activity [10]. Mah SH and his colleagues found that aqueous extract of Polygonum chinense L. had good effect 
in inhibiting inflammatory response [11]. Meanwhile, Ilex rotunda Thumb also had antibacterial and anti-inflammatory activities [12]. Bacterial infection and inflammatory are two main pathogenesis of PID; however, it is yet to be determined whether FYCWYC maintains strong antibacterial and anti-inflammatory activities and has some effects on PID. Pharmacological research of FYCWYC in lab may impart complete understanding of FYCWYC practice and more extensive application in China. In this study, we would observe preventive effect of FYCWYC on PID in rat model and further evaluates its pharmacological effect.

\section{Materials and Methods}

2.1. Materials. Escherichia coli (ATCC25922) and Staphylococcus aureus (ATCC25923) were purchased from American Type Culture Collection. Ultrapure water was obtained from a Milli-Q water purification system (Millipore, MA, USA). Detection kits for IL- $1 \beta$, TNF- $\alpha$, MCP-1, caspase-3, and IL6 were obtained from Nanjing Jiancheng Bioengineering Institute (Nanjing, China). NF- $\kappa \mathrm{B}, \mathrm{I} \kappa \mathrm{B}$, proliferating cell nuclear antigen (PCNA), Bcl-2, Bax, and $\beta$-actin antibodies were obtained from Cell Signaling Technologies, MA, USA. SP600125 (purity: 98\%) was purchased from Sigma-Aldrich Company (St. Louis, Missouri, USA).

2.2. Preparation of FYCWYC. Euphorbia hirta L., Polygonum chinense L., and Ilex rotunda Thunb were purchased from Zaolutang Co. (Xian, China) and identified botanically by Professor Haifeng Tang (Department of Natural Medicine, School of Pharmacy, Fourth Military Medical University). Three voucher specimens for these three herbs were deposited at the Department of Natural Medicine, School of Pharmacy, Fourth Military Medical University. All herbs (Euphorbia hirta L.:Polygonum chinense L.: Ilex rotunda Thunb $=4: 2: 1$ ) were cut into pieces and soaked for $20 \mathrm{~min}$ in purified water ( 8 volumes) and then boiled and decocted for 20 min over a low heat. Repeat this process for two times; the decoction was homogenized and concentrated.

2.3. Rat PID Model Construction and Animal Treatment. Sprague Dawley (SD) rats, female, weighing 180-220 g, were purchased from the Experimental Animal Center of the Fourth Military Medical University. The experimental procedures were approved by the Ethics Committee for Animal Experimentation. All experiments were performed according to the National Institute of Health Guide for the Care and Use of Laboratory Animals (NIH Publications number 80-23, revised 1996) and the Guidelines for Animal Experimentation of the Fourth Military Medical University. $12 \mathrm{~h}$ dark/light cycles under a temperature of $22-24^{\circ} \mathrm{C}$ and a relative humidity of $60 \%-65 \%$ standard conditions were kept. SD rats were divided into six groups randomly $(n=8)$ : control group (normal rats), PID group (rats infected by multipathogen), FYCWYC low-dose group (L, $0.6 \mathrm{~g} / \mathrm{kg}$ ), medial-dose group $(\mathrm{M}, 1.2 \mathrm{~g} / \mathrm{kg})$, high-dose group $(\mathrm{H}, 2.4 \mathrm{~g} / \mathrm{kg})$, and aspirin group (A, $2 \mathrm{mg} / \mathrm{kg}$ ).
Rats were fed 7 days to adapt to the environment and then injected with progesterone $(10 \mathrm{mg})$ subcutaneously. After these treatments, the PID model was constructed according to the methods reported previous [13]. Briefly, multipathogen solution which was composed of $U$. urealyticum $\left(1 \times 10^{8} \mathrm{ccu} / \mathrm{ml}\right)$ and $E$. coli $\left(1 \times 10^{8} \mathrm{ccu} / \mathrm{ml}\right)$ was prepared, and then absorbable gelatin sponge was immersed with $0.125 \mathrm{ml}$ microbe-mixing solution. Multipathogen solution was inserted into rats' upper genital tract except control group, and then the rats were forced to stand upside down for 3 minutes. Four-time infections were conducted during 2 days. Microbe-free gelatin sponges with saline were inserted into control group rats. To detect the $U$. urealyticum and $E$. coli in vaginal swab samples, mycoplasma detection kit and TDR-300B automatic microbe analysis system (Tiandiren, Changsha, China) were used. FYCWYC and aspirin were given to rats orally for 8 days from the first infection. Eight days later, the rats were anesthetized with pentobarbital $(30 \mathrm{mg} / \mathrm{kg}$ ) subcutaneously. For the future experiments, uterus and fallopian tubes from experiment rats were collected and restored at $-20^{\circ} \mathrm{C}$. After these experiments finished, the rats were sacrificed by decapitation.

2.4. Histological Evaluation. For histological evaluation, the left uterus and fallopian tube of experiment rats were collected and cut into $2 \mu \mathrm{m}$ sections, fixed by buffered $10 \%$ formaldehyde for $24 \mathrm{~h}$, dehydrated in alcohol, cleared in xylol, embedded in paraffin, and stained with hematoxylin-eosin at $\mathrm{pH}$ 3.5. Each slide was observed and evaluated randomly in 10 aleatory areas under a low-power microscopy field $(\times 100)$ by two experienced pathologists.

2.5. Enzyme-Linked Immunosorbent Assay (ELISA). Uterus and fallopian tubes in experiment rats were collected and weighed and then added to physiologic saline at the ratio of $1: 5(\mathrm{w} / \mathrm{v})$ and then homogenized. The levels of C-reactive protein (CRP), interleukin-1 $\beta$ (IL-1 $\beta$ ), interleukin-6 (IL6 ), and tumor necrosis factor-a (TNF-a) in homogenate were measured by ELISA kits (Nanjing Jiancheng, Nanjing, China). The protein levels in tissue were measured by bicinchoninic acid (BCA) protein assay kit. The concentrations of the inflammatory cytokines were expressed as $\mu \mathrm{g} / \mathrm{g}$ protein of homogenate.

2.6. Western Blotting. The upper genital tract tissue samples were homogenized and lysed in ice-cold RIPA lysis buffer, mixed with SDS-PAGE sample buffer $(2 \mathrm{x})$, boiled for $10 \mathrm{~min}$, and then resolved by $10 \%$ SDS-PAGE being transferred onto polyvinylidene difluoride (PVDF, Millipore, Billerica, MA, USA) membranes. Blots were blocked at $37^{\circ} \mathrm{C}$ for $60 \mathrm{~min}$ with $5 \%$ nonfat dry milk and then reacted with properly diluted monoclonal antibodies $(1: 1000)$ including NF- $\kappa \mathrm{B}$ p65, I $\kappa$ B, Akt, P-Akt, P-P38, P38, P-JNK, JNK, PCNA, $\beta$ actin, Bax, and Bcl-2. The membranes were washed and incubated with horseradish peroxidase-conjugated goat antimouse IgG antibodies $(1: 1,000$; Santa Cruz Biotechnology) at $37^{\circ} \mathrm{C}$ for $1 \mathrm{~h}$, followed by enhanced chemiluminescence reaction (Pierce Biotechnology, USA). 


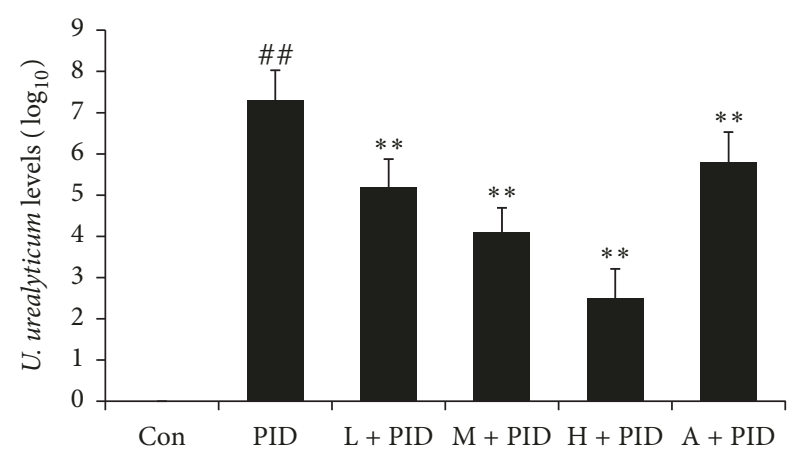

(a)

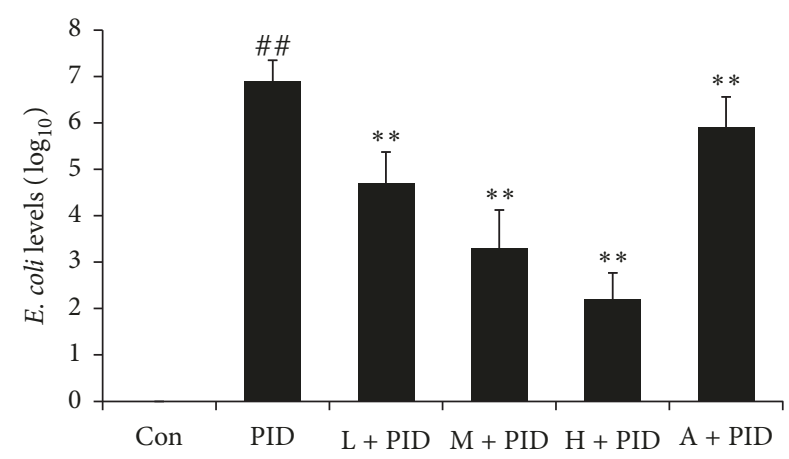

(b)

FIgURE 1: The antibacterial activity of FYCWYC was tested by pathogen examination. (a) The effects of FYCWYC on the U. urealyticum levels in the vaginal swab samples. (b) The effects of FYCWYC on the E. coli levels in the vaginal swab samples. Data were presented as the means \pm standard error $(n=8) .{ }^{\# \#} P<0.01$ versus control group, ${ }^{* *} P<0.01$ versus PID group.

2.7. Determination of Caspase-3 Activity. A fluorometric assay kit was used to measure the caspase-3 activity, and the protocol was according to the manufacturer. In brief, upper genital tract tissues of experiment rats were lysed in $50 \mu \mathrm{l}$ ice-cold lysis buffer for $30 \mathrm{~min}$ on ice and then centrifuged at $12000 \mathrm{~g}$ at $4^{\circ} \mathrm{C}$ for $10 \mathrm{~min}$. The protein levels in tissue were measured by BCA protein assay kit. The resulting fluorescence at $400 \mathrm{~nm}$ was measured by a microplate reader (Fluoroskan Ascent, Thermo Labsystems, Waltham, MA).

2.8. Statistical Analyses. The statistical data are presented as the means \pm standard deviation (SD) of the indicated numbers of samples. The differences between two data sets were evaluated using Student's $t$-test by the SPSS 18.0 statistical software (SPSS, USA). One-way analysis of variance (ANOVA) was used to compare the difference between more than two data sets. $P$ values of $<0.05$ were considered to indicate a statistically significant difference.

\section{Results}

3.1. Pathogens and Histological Examination. To test the antibacterial activity of FYCWYC, pathogen examination was used. The results showed that vaginal swab samples from control rats showed negative results for U. urealyticum and $E$. coli, but vaginal swab samples from PID rats showed positive results for both, indicating successful infection in PID rats (Figure 1). The vaginal swab samples from FYCWYC treatment rats showed less U. urealyticum and E. coli, indicating that FYCWYC had antibacterial activity.

Histological evaluation results were showed in Figure 2. In PID group rats, the uterus and fallopian tube were infiltrated by mass inflammatory cells, including neutrophils and lymphocytes. These results indicated that there was inflammation response in the upper genital tract. Compared with PID group, FYCWYC significantly decreased the inflammatory cells and mast cells infiltration in upper genital tract (Figure 2(a)). And the semiquantitative results also showed the significant differences between PID group and FYCWYC group on inflammatory cells (Figure 2(b)) and mast cells (Figure 2(c)) numbers.
3.2. FYCWYC Inhibited the Inflammatory Response in Rat PID Model. To observe the inflammatory response, the IL-1 $\beta$, IL6 , CRP-1, and TNF- $\alpha$ levels in PID rats were measured by ELISA kits. As the results showed in Figure 3, compared with control group, IL-1 $\beta$, IL-6, CRP-1, and TNF- $\alpha$ levels were significantly increased in PID group, and FYCWYC significantly inhibited the production of these cytokines in rats. These results indicated that there was inflammatory response in PID rats and FYCWYC had anti-inflammatory effects.

3.3. FYCWYC Inhibited the Apoptosis in PID Model. To determine the mechanism of FYCWYC, apoptosis in upper genital tract was measured. As the results showed in Figure 4, the caspase- 3 levels and the Bax expression were significantly increased in PID rats and the Bcl-2 expression was significantly decreased compared with control group. Compared with PID group, the caspase- 3 levels and the Bax expression were significantly decreased and the Bcl-2 expression was significantly increased. These results indicated that apoptosis was induced by bacterial infection and FYCWYC had some effects on apoptosis.

3.4. FYCWYC Inhibited the Expression of NF- $\kappa B$ and $I \kappa B-\alpha$ in PID Model. To make clear the potential anti-inflammatory mechanism of FYCWYC, the expression levels of NF- $\kappa$ B p 65 and $\mathrm{I} \kappa \mathrm{B}-\alpha$ in nuclear and/or cytoplasm were determined by Western blotting. In PID group, NF- $\kappa \mathrm{B}$ p 65 was translocated from cytoplasm to cell nucleus, and phospho-I $\kappa \mathrm{B}-\alpha$ level in cytoplasm was more than that in control groups. In FYCWYC treatment groups, more NF- $\kappa$ B p 65 was distributed in cytoplasm, indicating that FYCWYC could inhibit the translation of NF- $\kappa$ B p65 (Figure 5).

3.5. FYCWYC Inhibited the Phosphorylation of JNK Pathway. To further study the upstream of Nf- $\kappa \mathrm{B}$, the phosphorylation levels of Akt, p38 MAPK, and JNK were detected by Western blotting. As shown in Figure 6, we found that the phosphorylation levels of Akt, p38 MAPK, and JNK were increased in PID rats. Treatment with FYCWYC inhibited the increase of the JNK phosphorylation level. However, 


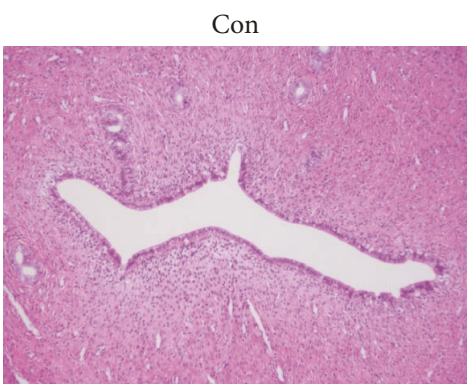

$\mathrm{M}+\mathrm{PID}$
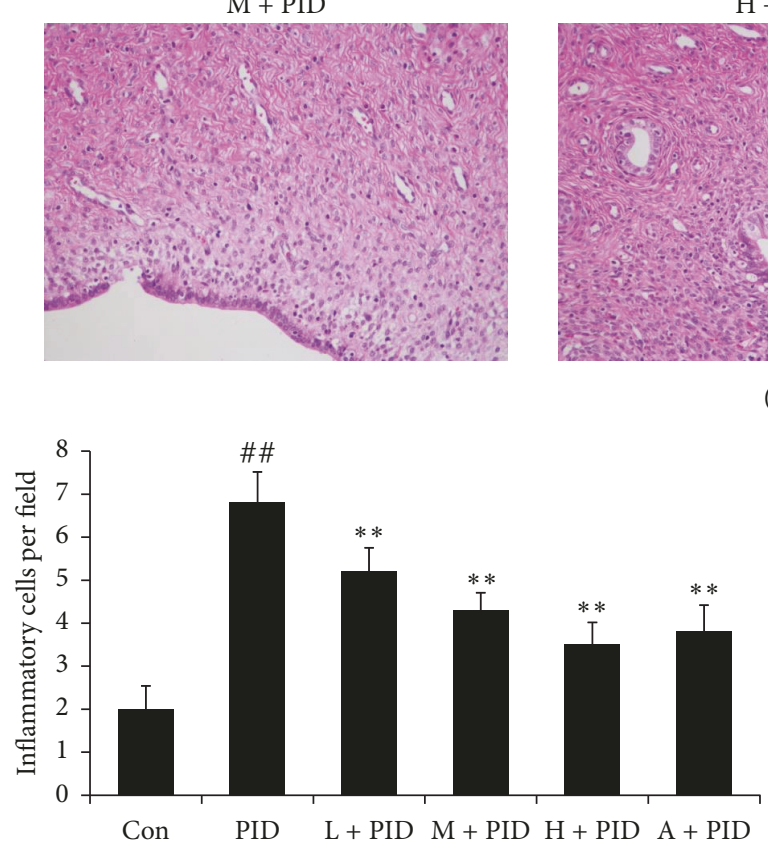

(b)

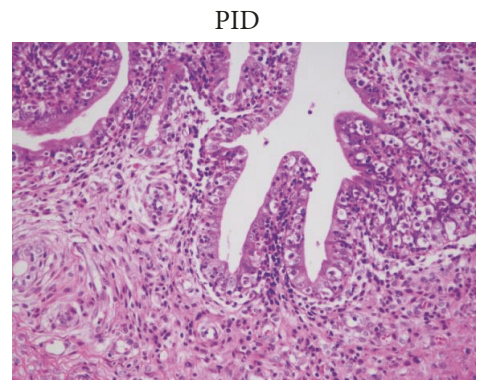

$\mathrm{H}+\mathrm{PID}$

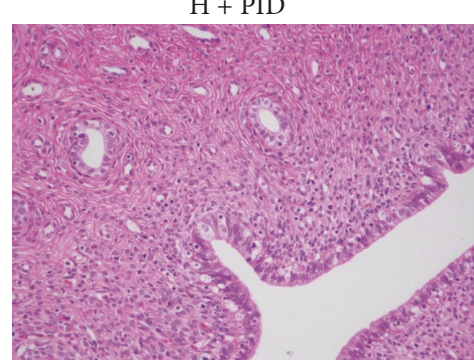

(a)

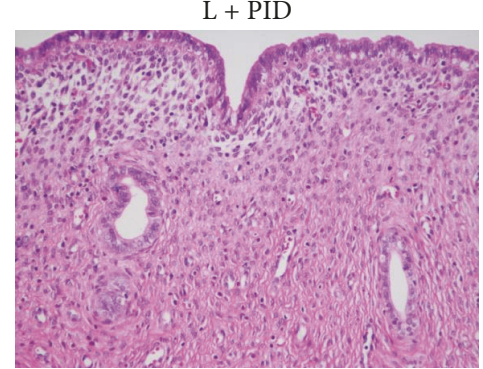

A + PID

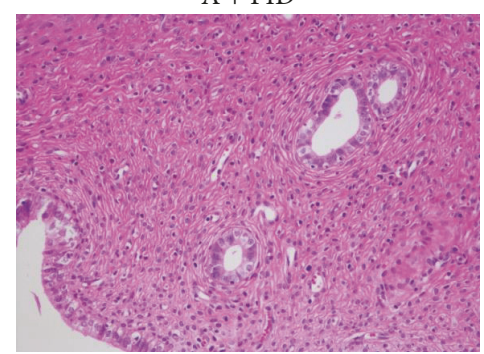

(c)

FIGURE 2: Effect of FYCWYC on infiltration of neutrophils and lymphocytes in uterus and fallopian tube after pathogenic infection. (a) Representative micrographs of uterus and fallopian. (b) Inflammatory cells numbers in the observed fields. (c) Mast cells numbers in the observed fields. Data were presented as the means \pm standard error $(n=8) .{ }^{\# \#} P<0.01$ versus control group, ${ }^{* *} P<0.01$ versus PID group.

FYCWYC had no effect on the phosphorylation levels of Akt and $\mathrm{p} 38$. These results suggested that FYCWYC inhibited the infection-induced NF- $\kappa \mathrm{B}$ translocation via the inhibition of JNK.

3.6. FYCWYC Inhibited the Expression of NF- $\mathrm{KB}$ through JNK. Next, the JNK pathway was examined to investigate whether it was involved in the protective roles of FYCWYC in PID model. SP600125, a phospho-JNK inhibitor, was used to inhibit the phosphorylation levels of JNK (Figure 7(a)). The Western blotting results indicated that SP600125 inhibited IkB-a phosphorylation, reduced the translocation of NF- $\kappa \mathrm{B}$ from cytoplasm to nuclear, and thus decreased the NF- $\kappa \mathrm{B}$ activity. SP600125 also decreased the level of caspase-3 and IL-6 induced by infection (Figures 7(b) and 7(c)). These results indicated that the inhibition of phospho-JNK suppressed the activation of the NF-kB pathway and protected the cells from apoptosis and inflammation, which was similar to the role of FYCWYC.

\section{Discussion}

PID is a popular inflammation and infection in the women's upper genital tract, especially involving the uterus and fallopian tube. In some regions of US, PID is the most common gynecological reason in the hospital, about 18/10,000 recorded hospital discharges [14]. Among women with PID, about $40 \%$ develop into chronic pain, $20 \%$ become infertile, and $1 \%$ have an ectopic pregnancy [15]. In clinic, several pathogens which include U. urealyticum, E. coli, and some other Gram-negative and Gram-positive bacteria can lead to PID [16-19]. In this study, U. urealyticum and E. coli mixed solutions were used to promote inflammation in upper genital tract to simulate the PID model.

In clinic, broad spectrum antibiotics which are associated with high rates of short term improvement were always used to treat PID; however, there is an increased risk of tubal damage leading to chronic pelvic pain and infertility [20]. Nonsteroid anti-inflammatory drugs (NSAIDs) were also used in treating gynecological diseases, but NSAIDs play a 


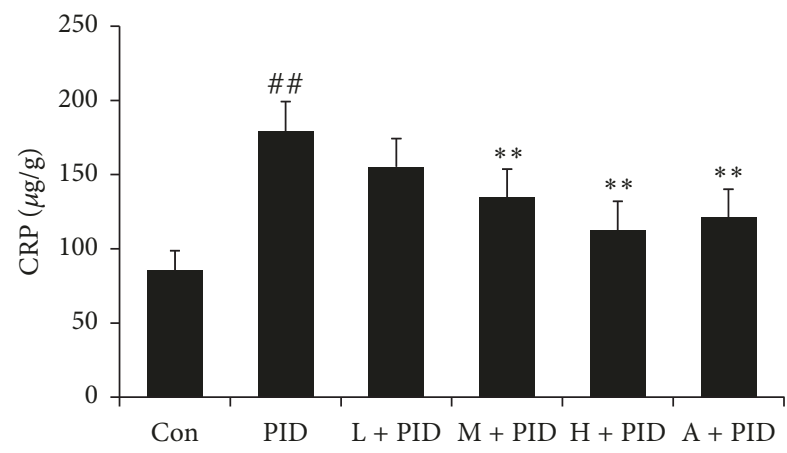

(a)

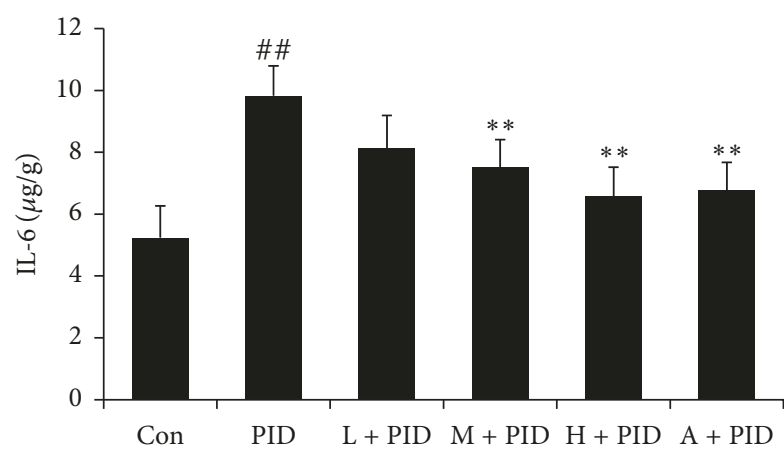

(c)

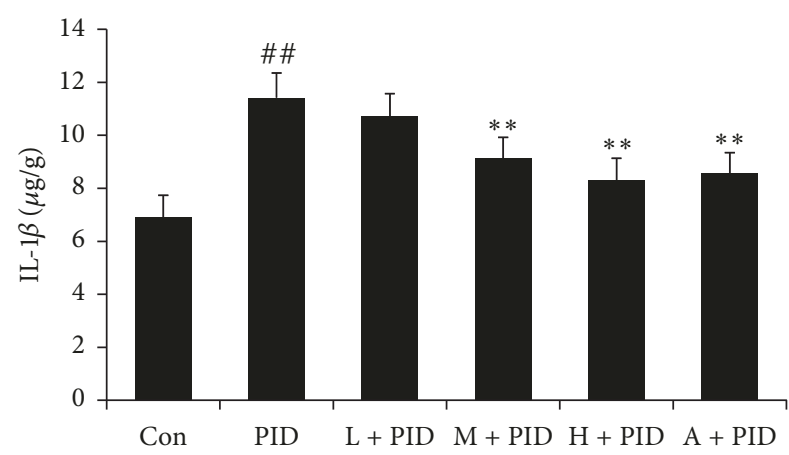

(b)

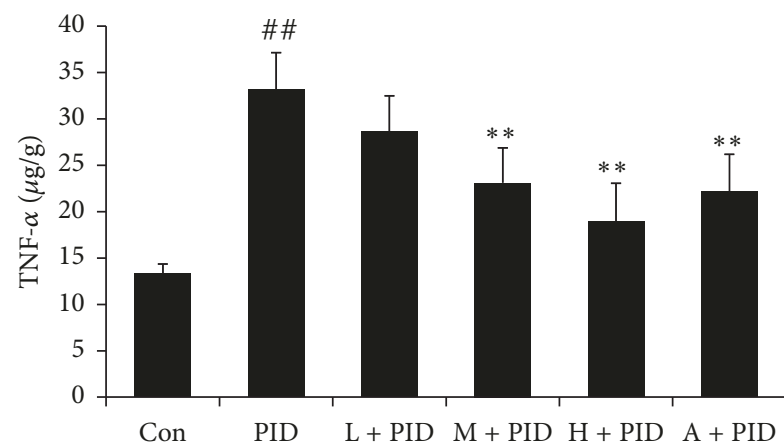

(d)

FIGURE 3: Effect of FYCWYC on the inflammatory response in PID model. (a) Effect of FYCWYC on CRP level in the upper genital tract after pathogenic infection. (b) Effect of FYCWYC on IL-1 $\beta$ level in the upper genital tract after pathogenic infection. (c) Effect of FYCWYC on IL-6 level in the upper genital tract after pathogenic infection. (d) Effect of FYCWYC on TNF- $\alpha$ level in the upper genital tract after pathogenic infection. Data were presented as the means \pm standard error $(n=8) .{ }^{\#} P<0.01$ versus control group, ${ }^{* *} P<0.01$ versus PID group.

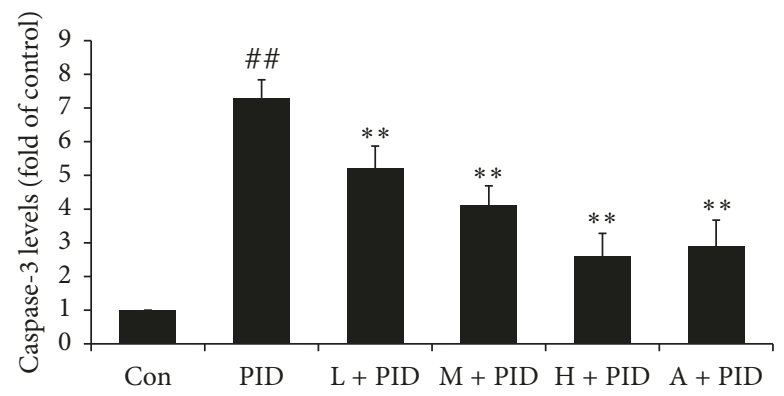

(a)
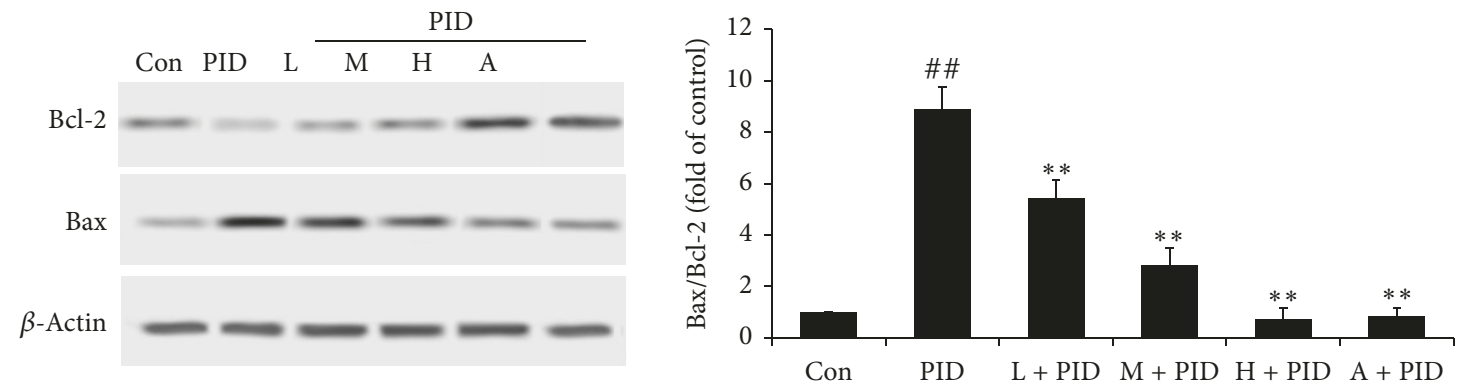

(b)

FIGURE 4: Effect of FYCWYC on the apoptosis in PID model. (a) Caspase-3 levels in the upper genital tract after pathogenic infection. (b) $\mathrm{Bcl}-2$ and Bax protein expression levels in the upper genital tract after pathogenic infection. Data were presented as the means \pm standard error $(n=8) .{ }^{\#} P<0.01$ versus control group, ${ }^{* *} P<0.01$ versus PID group. 

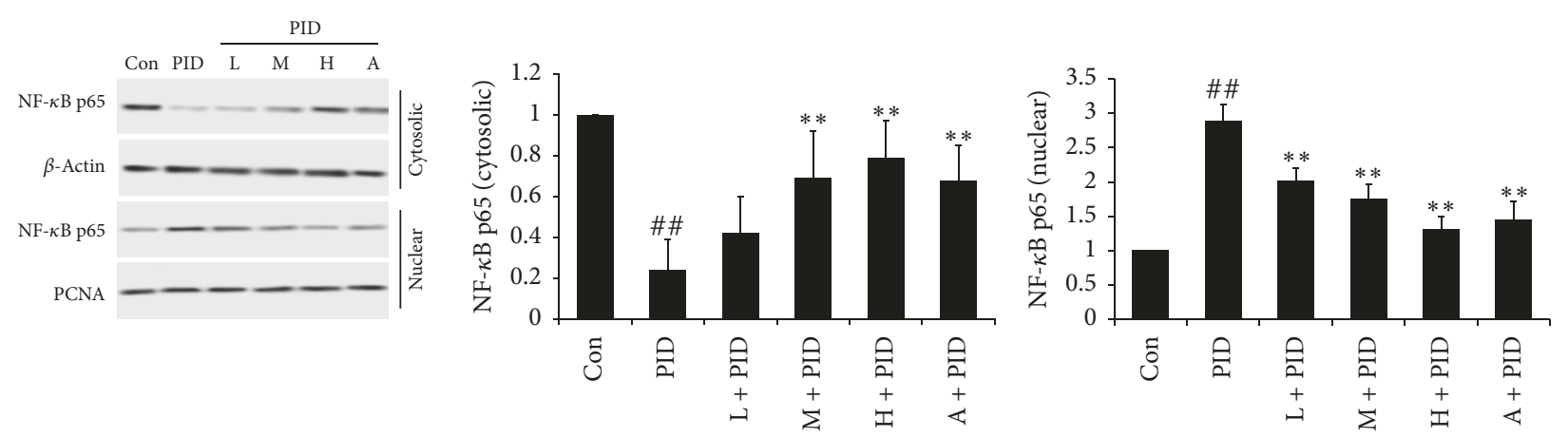

(a)
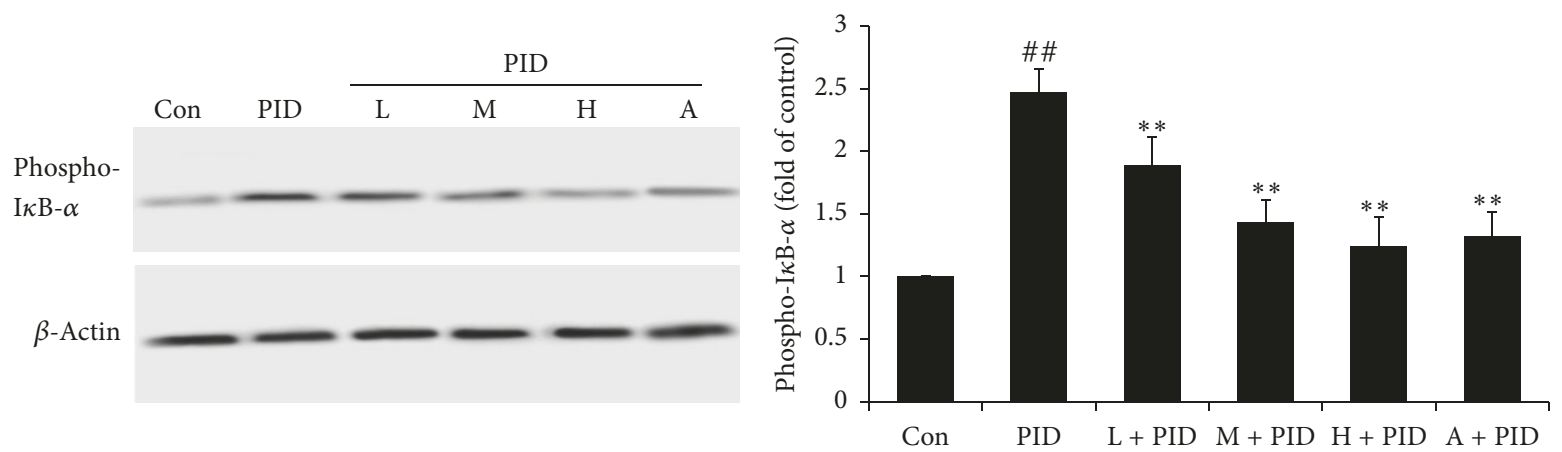

(b)

Figure 5: Effect of FYCWYC on the NF- $\kappa$ B p65 and I $\kappa$ B- $\alpha$ expression in PID model. (a) NF- $\kappa$ B p65 levels in cytoplasm and nuclear were tested by Western blotting. (b) Phospho-I $\kappa \mathrm{B}-\alpha$ in cytoplasm was tested by Western blotting. Data were presented as the means \pm standard error. ${ }^{\# \#} P<0.01$ versus control group, ${ }^{* *} P<0.01$ versus PID group.

limited role in the treatment once SPID forms, because of pelvic adhesion and fibrosis [21]. Long time and excessive use of NSAIDs also had obvious adverse effects, mainly involving ulcer, dyspepsia, and gastrointestinal bleeding, renal impairment, drug resistance, and weakened immunity $[22,23]$. TCM had been used for thousands of years. Based on the thoughts of TCM, it is not only improving the main symptoms, but also addressing associated symptoms [24]. TCM has gained satisfactory effects in treating PID in the past years. In present study, we tried to clarify the protective effects of FYCWYC in treating PID and illuminate the possible mechanism.

In the present antibacterial examination, FYCWYC showed antibacterial activity in vivo. Many neutrophils and lymphocytes were found in the epithelium of upper genital tract after chronic pathogen infections, and FYCWYC significantly decreased the inflammatory cells infiltration in upper genital tract, indicating that FYCWYC inhibited tissue damage through inhibiting inflammation.

After pathogen infection in the body and recognition of immunogens by the TLRs, inflammatory response was initiated and propagated, and among this process, proinflammatory cytokines play essential roles [25-27]. As we known, IL- $1 \beta$ and IL- 6 were two main proinflammatory cytokines. In upper genital tract of PID patients, elevated IL- $1 \beta$ could increase IL- 6 level and in turn suppress the IL- $1 \beta$ synthesis in the second phase of the immune response $[28,29]$. In upper genital tract, these proinflammatory cytokines can induce chemokines (CXCL-1, MCP-1) production which lead to hematopoietic immune cells recruitment and stimulate the proliferation and activation of leukocyte. Thus, neutrophils were activated and released inflammatory several cytokines and chemokines, which further intensify the inflammatory response [30]. In this study, we observed that the cytokines levels (IL-1 $\beta$, IL-6, CRP-1, and TNF- $\alpha$ ) in upper genital tract of PID rats were elevated, indicating inflammatory response was induced. Besides, treatment with FYCWYC significantly decreased these cytokines levels in a dose-dependent manner, suggesting a potent anti-inflammatory effect of FYCWYC.

Cell death can be found after necrosis and/or apoptosis. Necrosis and apoptosis were always accompanied by an aggressive inflammatory response and regulated many genetic and biochemical process [31]. As a response to infection, cell apoptosis has been observed in many pathogens infection [32, 33]. The caspase family of proteases plays important role in many aspects of apoptosis [34]. Caspase3, a key enzyme in the downstream of apoptotic pathway, promoted apoptosis factors and then leads to apoptosis through caspase-3-mediated signaling pathways when it is active [35]. In this study, we found that caspase-3 levels were increased in PID group; however, in FYCWYC treatment groups, caspase-3 levels were significantly decreased. During infection in cells, Bcl-2 family proteins play important roles in the anti- and proapoptotic processes [36, 37]. In the 

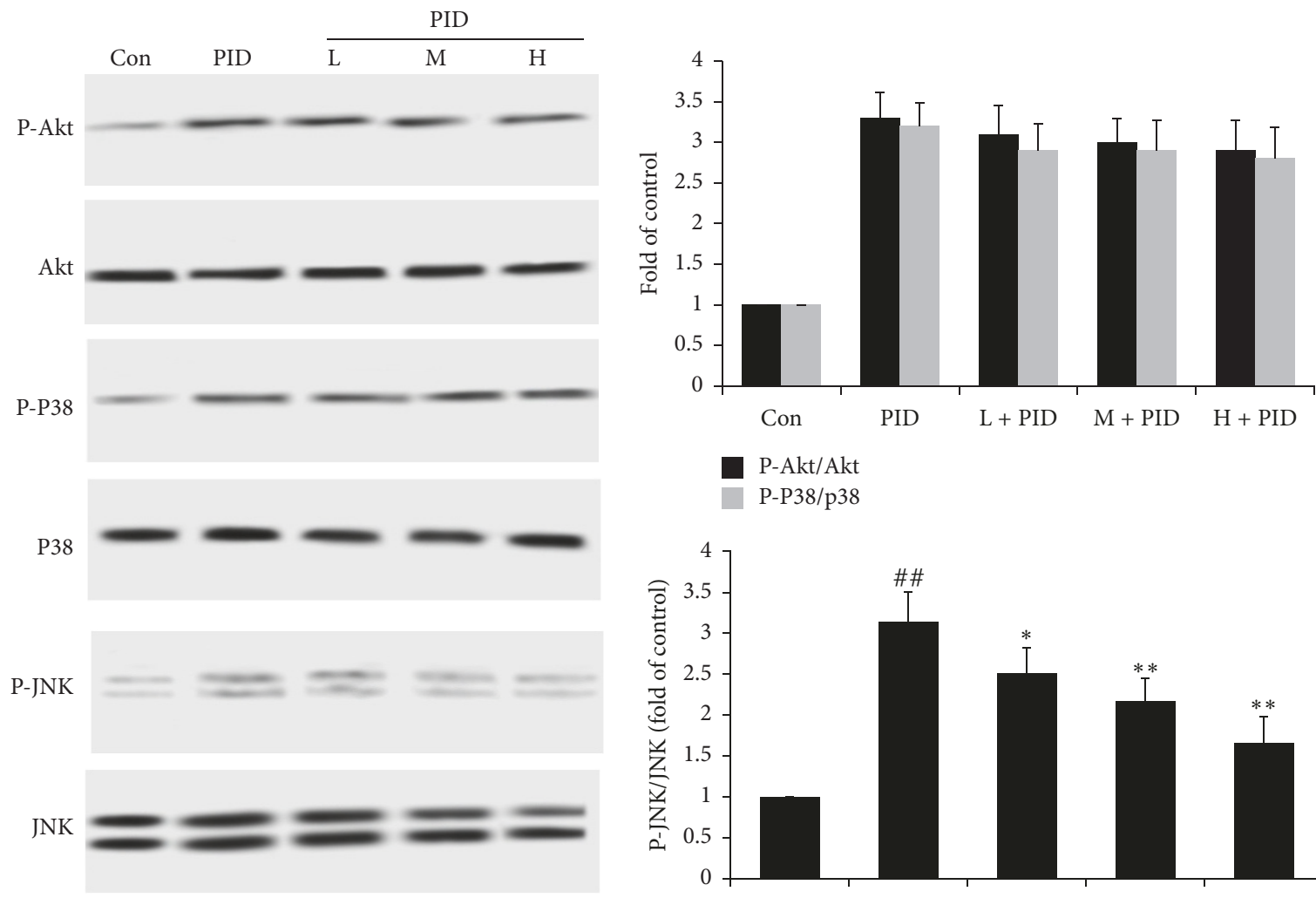

P-Akt/Akt

P-P38/p38

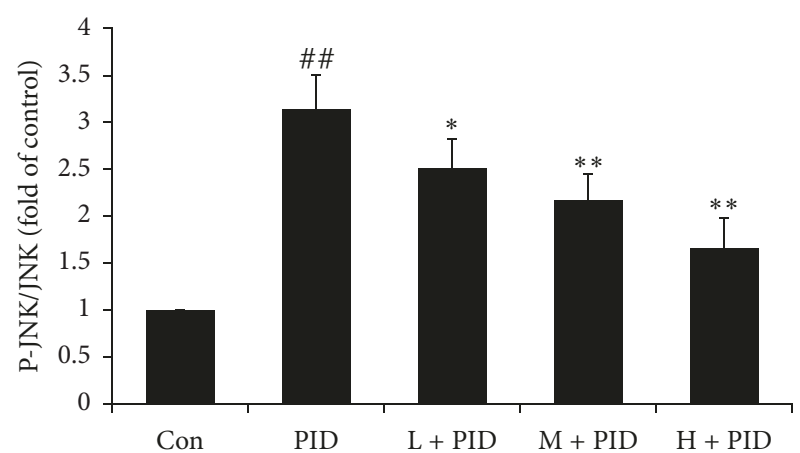

FIGURE 6: The effect of FYCWYC on the upstream of NF-kB pathway in PID model. The Akt phosphorylation, JNK phosphorylation, and p38 phosphorylation levels were tested by Western blotting. Data were presented as the means \pm standard error. ${ }^{\# \#} P<0.01$ versus control group, ${ }^{*} P<0.05,{ }^{* *} P<0.01$ versus PID group.

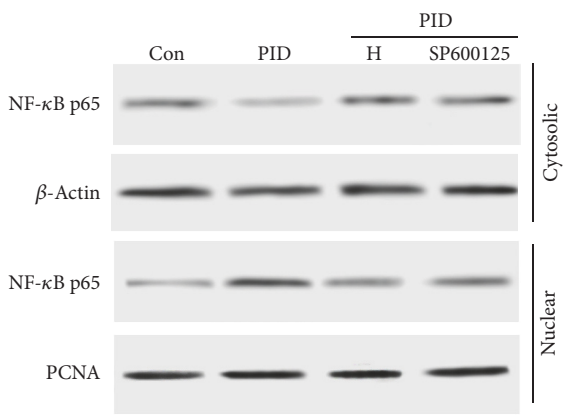

(a)

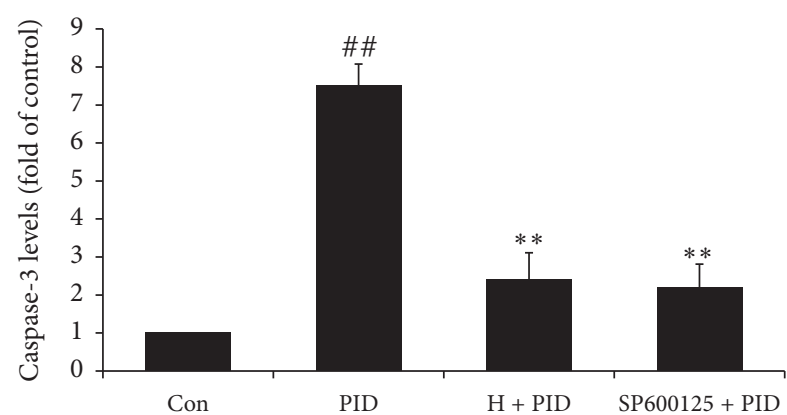

(b)

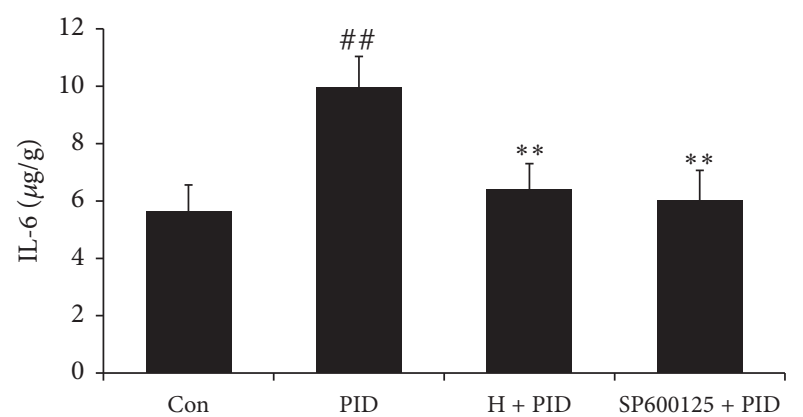

(c)

FIGURE 7: Inhibition of JNK phosphorylation by treatment by FYCWYC. (a) Rats were given an inhibitor of phospho-JNK, SP600125 $(30 \mathrm{mg} / \mathrm{kg})$ through intragastric administration. (a) NF- $\kappa \mathrm{B}$ p65 levels in cytoplasm and nuclear were tested by Western blotting. (b) Levels of caspase- 3 were measured by caspase- 3 kit. (c) IL- 6 levels were measured by IL- 6 kit. Data were presented as the means \pm standard error. ${ }^{\# \#} P<0.01$ versus control group, ${ }^{* *} P<0.01$ versus PID group. 
present study, Bax and Bcl-2 were measured, and we found that Bax expression was increased and Bcl-2 expression was decreased. However, FYCWYC reversed those changes. These results suggested that apoptosis was induced in PID rats and inhibited by FYCWYC treatment.

$\mathrm{NF}-\kappa \mathrm{B}$, a crucial and ubiquitous transcriptional factor, plays important roles in regulating the transcription of several intercellular signaling pathways which was involved in inflammatory and immune responses [38]. Under normal condition, NF- $\kappa \mathrm{B}$ exists in the cytoplasm in an inactive complex with $\mathrm{I} \kappa \mathrm{B}$ [39]. Upon infection, TLRs recognized pathogens and resulted in the phosphorylation and degradation of $\mathrm{I} \kappa \mathrm{B}$; then $\mathrm{NF}-\kappa \mathrm{B}$ is free to translocate into the nucleus and promote the expression of many inflammatory mediators, such as IL-1 $\beta$ and IL-6 [40]. Those inflammatory mediators further activate NF- $\kappa \mathrm{B}$ and subsequently promote more proinflammatory mediators production [41]. Some pathogens that had been reported could activate NF- $\kappa \mathrm{B}$, including M. genitalium, M. fermentans, and M. pneumonia [42-44]. In uterus and fallopian tube, NF- $\kappa \mathrm{B}$ p65 and $\mathrm{I} \kappa \mathrm{B}$ are representative members of $\mathrm{NF}-\kappa \mathrm{B}$ and $\mathrm{I} \kappa \mathrm{B}$ family, respectively [45]. As showed in our results, multi-infection of pathogens caused the translation of NF- $\kappa \mathrm{B}$ p 65 in uterus tissues. In the rat PID model, FYCWYC might exert its antiinflammatory effects through blocking the NF- $\kappa$ B pathway activation.

Many signaling pathways act as the upstream regulation protein of NF- $\kappa \mathrm{B}$, like PI3K/Akt and MAPK signaling pathways. PI3K/AKT signaling pathway regulates a series of changes through lots of proteins including NF- $\kappa \mathrm{B}$ [46]. When PI3K/Akt was phosphorylated by some factors, IkBa phosphorylation was induced and then leads to NF-kB activation [47]. It has been reported that MAPK, especially c-Jun $\mathrm{NH}(2)$-terminal kinase (JNK), induces NF-kB activation at some conditions [48]. A variety of cellular stresses could induce and activate JNK and p38 MAPK, such as inflammatory cytokines [49]. JNK and p38 MAPK activation contributes to cell apoptosis and cell death [50]. Some researchers also found that virus infection could induce the activation of JNK and p38 MAPK and cause injury to patients [51]. However, there was little report about the relationship between MAPK and NF-kB in PID. In this study, we found that infection induced phosphorylation of AKT, JNK, and p38 MAPK, and FYCWYC only decreased JNK phosphorylation. An inhibitor of phospho-JNK, SP600125, was used to confirm its role in PID. We found that SP600125 inhibited the translation of NF- $\kappa \mathrm{B}$ p 65 and cell inflammation and apoptosis induced by infection.

\section{Conclusions}

In this study, the preventive effect of FYCWYC against PID was found in a rat model, including reducing excessive production of inflammatory cytokines and inhibiting the infiltration of neutrophils and lymphocytes. Further, we also found that the potential mechanism of this effect might be associated with the suppression of JNK-NF- $\kappa \mathrm{B}$ pathway. The data from this study provided scientific foundation for the clinical uses of FYCWYC in alleviating PID infection and treating inflammatory disorders.

\section{Conflicts of Interest}

The authors declare that they have no conflicts of interest.

\section{Authors' Contributions}

Yao Li and Yang Liu contributed equally to the work.

\section{Acknowledgments}

This work was supported by the grant from the Social Development of Shaanxi Province Key Project (no. S2017-ZDYFZDXM-SF-0131), Science and Technology Innovation Project of Shaanxi province in China (no. 2015SF2-08-01), and Key Research Laboratory of Traditional Chinese Medicine and Natural Medicine in Shaanxi Province (no. 2015-164).

\section{References}

[1] J. H. Barash, C. Hillson, and E. Buchanan, Suaray M: Pelvic Inflammatory Disease, Humana Press, 2013.

[2] W. Zou, Z. Xiao, X. Wen et al., "The anti-inflammatory effect of Andrographis paniculata (Burm. f.) Nees on pelvic inflammatory disease in rats through down-regulation of the NF-KB pathway," BMC Complementary and Alternative Medicine, vol. 16, no. 1, article no. 483, 2016.

[3] R. D. V. Miguel, M. Chivukula, U. Krishnamurti et al., "Limitations of the criteria used to diagnose histologic endometritis in epidemiologic pelvic inflammatory disease research," Pathology - Research and Practice, vol. 207, no. 11, pp. 680-685, 2011.

[4] K. Deb, M. M. Chaturvedi, and Y. K. Jaiswal, "Comprehending the role of LPS in Gram-negative bacterial vaginosis: ogling into the causes of unfulfilled child-wish.," Archives of Gynecology and Obstetrics, vol. 270, no. 3, pp. 133-146, 2004.

[5] I. Bernstein-Hanley, J. Coers, Z. R. Balsara, G. A. Taylor, M. N. Starnbach, and W. F. Dietrich, "The p47 GTPases Igtp and Irgb10 map to the Chlamydia trachomatis susceptibility locus Ctrq-3 and mediate cellular resistance in mice," Proceedings of the National Acadamy of Sciences of the United States of America, vol. 103, no. 38, pp. 14092-14097, 2006.

[6] I. M. Sheldon, D. E. Noakes, A. N. Rycroft, D. U. Pfeiffer, and H. Dobson, "Influence of uterine bacterial contamination after parturition on ovarian dominant follicle selection and follicle growth and function in cattle," Reproduction, vol. 123, no. 6, pp. 837-845, 2002.

[7] D. Rui, D. Fuhrich, and J. D. C. Ross, "A review of antibiotic therapy for pelvic inflammatory disease," International Journal of Antimicrobial Agents, vol. 46, no. 3, pp. 272-277, 2015.

[8] R. Yang, Clinical observation of Feiyang Changweiyan table treat for irritable bowel syndrome, China Modern Medicine, 2012.

[9] M.-C. Lanhers, J. Fleurentin, P. Dorfman, F. Mortier, and J.-M. Pelt, "Analgesic, antipyretic and anti-inflammatory properties of Euphorbia hirta," Planta Medica, vol. 57, no. 3, pp. 225-231, 1991.

[10] A. Annamalai, V. L. P. Christina, D. Sudha, M. Kalpana, and P. T. V. Lakshmi, "Green synthesis, characterization and antimicrobial activity of Au NPs using Euphorbia hirta L. leaf 
extract," Colloids and Surfaces B: Biointerfaces, vol. 108, pp. 6065, 2013.

[11] P. Y. Ouyang, C. X. Zhu, G. X. Chen, and L. F. Deng, Preliminary Research of Antimicrobial Activity of the Extract from Polygonum Chinense Linn, Chemistry \& Bioengineering, 2012.

[12] M. Kim, S. Choi, K. Park et al., "Anti-oxidative and antiinflammatory activities of caffeoyl derivatives from the barks of Ilex rotunda," Planta Medica, vol. 77, no. 12, 2011.

[13] W. Zou, X. Wen, Y. Zheng et al., "Metabolomic Study on the Preventive Effect of Patrinia scabiosaefolia Fisch on Multipathogen Induced Pelvic Inflammatory Disease in Rats," Evidence-Based Complementary and Alternative Medicine, vol. 2015, Article ID 170792, 2015.

[14] M. Y. Sutton, M. Sternberg, A. Zaidi, M. E. St. Louis, and L. E. Markowitz, "Trends in pelvic inflammatory disease hospital discharges and ambulatory visits, United States, 1985-2001," Sexually Transmitted Diseases, vol. 32, no. 12, pp. 778-784, 2005.

[15] T. Darville, "Pelvic inflammatory disease: Identifying research gapsvproceedings of a workshop sponsored by department of health and human services/ national institutes of health/ national institute of allergy and infectious diseases, november 3y4, 2011," Sexually Transmitted Diseases, vol. 40, no. 10, pp. 761767, 2013.

[16] World Health Organization and Department of Reproductive Health and Research, "Global incidence and prevalence of selected curable sexually transmitted infections: 2008," Reproductive Health Matters, vol. 20, no. 40, pp. 207-209, 2012.

[17] R. Quentin and R. Verdon, "Microbiologic basis of diagnosis and treatment of pelvic inflammatory disease," Journal de Gynécologie Obstétrique et Biologie de la Reproduction, vol. 41, no. 8, pp. 850-863, 2012.

[18] S. Saini, N. Gupta, A. Aparna, G. Batra, and D. R. Arora, "Role of anaerobes in acute pelvic inflammatory disease," Indian Journal of Medical Microbiology, vol. 21, no. 3, pp. 189-192, 2003.

[19] D. Z. Zhang, J. Y. Wen, W. C. Zhou, and W. U. Xiao-Yan, "Pathogenic Bacteria Distribution and Drug Resistance Isolated from Women with Pelvic Inflammatory Disease," Chinese Journal of Nosocomiology, 2009.

[20] C. Mitchell and M. Prabhu, "Pelvic Inflammatory Disease: Current Concepts in Pathogenesis, Diagnosis and Treatment," Infectious Disease Clinics of North America, vol. 27, no. 4, pp. 793-809, 2013.

[21] L. L. Fan, W. H. Yu, X. Q. Liu, Z. Cui, J. Ma, and C. P. Li, "A meta-analysis on effectiveness of acupuncture and moxibustion for chronic pelvic inflammatory disease," Acupuncture Research, vol. 39, no. 2, pp. 156-163, 2014.

[22] X. F. Cai, L. F. Chen, Z. L. Wang, and Y. E. Gu, "Effect of acupoint injection by Astragalus injection on local SIgA and pathomorphology changes in rats with chronic pelvic inflammatory disease," China Journal of Chinese Materia Medica, vol. 31, no. 16, pp. 1361-1364, 2006.

[23] J. A. Baron, S. Senn, M. Voelker et al., "Gastrointestinal adverse effects of short-term aspirin use: a meta-analysis of published randomized controlled trials," Drugs in R\&D, vol. 13, no. 1, pp. 9-16, 2013.

[24] D. Geng and R. J. Guo, “TCM symptoms of anxiety disorders studied based on modern literature," Journal of Beijing University of Traditional Chinese Medicine, vol. 36, no. 7, pp. 484-487, 2013.

[25] C. Tortorella, G. Piazzolla, M. Matteo et al., "Interleukin6 , interleukin- $1 \beta$, and tumor necrosis factor $\alpha$ in menstrual effluents as biomarkers of chronic endometritis," Fertility and Sterility, vol. 101, no. 1, pp. 242-247, 2014.

[26] J. G. Cronin, M. L. Turner, L. Goetze, C. E. Bryant, and I. M. Sheldon, "Toll-like receptor 4 and MYD88-dependent signaling mechanisms of the innate immune system are essential for the response to lipopolysaccharide by epithelial and stromal cells of the bovine endometrium," Biology of Reproduction, vol. 86, no. 2, article no. 51, 2012.

[27] M. L. Turner, J. G. Cronin, G. D. Healey, and I. M. Sheldon, "Epithelial and stromal cells of bovine endometrium have roles in innate immunity and initiate inflammatory responses to bacterial lipopeptides in vitro via Toll-like receptors TLR2, TLR1, and TLR6," Endocrinology, vol. 155, no. 4, pp. 1453-1465, 2014.

[28] W. Cheng, P. Shivshankar, Z. Li, L. Chen, I.-T. Yeh, and G. Zhong, "Caspase-1 contributes to Chlamydia trachomatisinduced upper urogenital tract inflammatory pathologies without affecting the course of infection," Infection and Immunity, vol. 76, no. 2, pp. 515-522, 2008.

[29] A. Trunov, O. Obukhova, O. Gorbenko, and A. Shvayk, "Trunova L: Cytokines, estradiol and progesterone in the plasma of women of reproductive age with pelvic inflammatory disease in remission," in Advances in Bioscience Biotechnology, vol. 4, pp. 727-730, Trunova L, Cytokines, 2013.

[30] S. Gasperini, R. Zambello, C. Agostini et al., "Impaired cytokine production by neutrophils isolated from patients with AIDS," AIDS, vol. 12, no. 4, pp. 373-379, 1998.

[31] E. Duvall and A. H. Wyllie, "Death and the cell," Trends in Immunology, vol. 7, no. 4, pp. 115-119, 1986.

[32] S. F. Fischer and G. Häcker, "Characterization of Antiapoptotic Activities of Chlamydia pneumoniae in Infected Cells," Annals of the New York Academy of Sciences, vol. 1010, pp. 565-567, 2003.

[33] S. F. Fischer, C. Schwarz, J. Vier, and G. Häcker, "Characterization of antiapoptotic activities of Chlamydia pneumoniae in human cells," Infection and Immunity, vol. 69, no. 11, pp. 71217129, 2001.

[34] Y. Shi, "Mechanisms of caspase activation and inhibition during apoptosis," Molecular Cell, vol. 9, no. 3, pp. 459-470, 2002.

[35] A. G. Porter and R. U. Jänicke, "Emerging roles of caspase-3 in apoptosis," Cell Death \& Differentiation, vol. 6, no. 2, pp. 99-104, 1999.

[36] M. J. Binnicker, R. D. Williams, and M. A. Apicella, "Infection of human urethral epithelium with Neisseria gonorhoeae elicits an upregulation of host anti-apoptotic factors and protects cells from staurosporine-induced apoptosis," Cellular Microbiology, vol. 5, no. 8, pp. 549-560, 2003.

[37] M. J. Binnicker, R. D. Williams, and M. A. Apicella, "Gonococcal porin IB activates NF- $\kappa$ B in human urethral epithelium and increases the expression of host antiapoptotic factors," Infection and Immunity, vol. 72, no. 11, pp. 6408-6417, 2004.

[38] M. Helenius, M. Hänninen, S. K. Lehtinen, and A. Salminen, "Aging-induced up-regulation of nuclear binding activities of oxidative stress responsive NF-kB transcription factor in mouse cardiac muscle," Journal of Molecular and Cellular Cardiology, vol. 28, no. 3, pp. 487-498, 1996.

[39] S. Ghosh, M. J. May, and E. B. Kopp, "NF- $\kappa$ B and rel proteins: evolutionarily conserved mediators of immune responses," Annual Review of Immunology, vol. 16, pp. 225-260, 1998.

[40] J. He, X. You, Y. Zeng, M. Yu, L. Zuo, and Y. Wu, "Mycoplasma genitalium-Derived Lipid-Associated Membrane Proteins Activate NF- $\kappa$ B through Toll-Like Receptors 1, 2, and 6 and 
CD14 in a MyD88-Dependent Pathway," Clinical and Vaccine Immunology, vol. 16, no. 12, pp. 1750-1757, 2009.

[41] V. Bours, G. Bonizzi, M. Bentires-Alj et al., "NF- $\kappa$ B activation in response to toxical and therapeutical agents: Role in inflammation and cancer treatment," Toxicology, vol. 153, no. 1-3, pp. 27-38, 2000.

[42] T. Into, K. Kiura, M. Yasuda et al., "Stimulation of human Tolllike receptor (TLR) 2 and TLR6 with membrane lipoproteins of Mycoplasma fermentans induces apoptotic cell death after NF$\kappa \mathrm{B}$ activation," Cellular Microbiology, vol. 6, no. 2, pp. 187-199, 2004.

[43] C. L. McGowin, M. Liang, D. H. Martin, and R. B. Pyles, "Mycoplasma genitalium-encoded MG309 activates NF- $\kappa$ B via toll-like receptors 2 and 6 to elicit proinflammatory cytokine secretion from human genital epithelial cells," Infection and Immunity, vol. 77, no. 3, pp. 1175-1181, 2009.

[44] T. Shimizu, Y. Kida, and K. Kuwano, "A dipalmitoylated lipoprotein from Mycoplasma pneumoniae activates NF- $\kappa$ B through TLR1, TLR2, and TLR6," The Journal of Immunology, vol. 175, no. 7, pp. 4641-4646, 2005.

[45] L. Dai, L. Gu, and W. Di, "MiR-199a attenuates endometrial stromal cell invasiveness through suppression of the IKK $\beta / \mathrm{nf}$ $\kappa \mathrm{b}$ pathway and reduced interleukin-8 expression," Molecular Human Reproduction, vol. 18, no. 3, Article ID gar066, pp. 136145, 2012.

[46] J. Chen, "Multiple signal pathways in obesity-associated cancer," Obesity Reviews, vol. 12, no. 12, pp. 1063-1070, 2011.

[47] M. G. Dilshara, K.-T. Lee, R. G. P. T. Jayasooriya et al., "Downregulation of NO and PGE2 in LPS-stimulated BV2 microglial cells by trans-isoferulic acid via suppression of PI3K/Akt-dependent NF- $\kappa \mathrm{B}$ and activation of Nrf2-mediated HO-1," International Immunopharmacology, vol. 18, no. 1, pp. 203-211, 2014.

[48] Y. Pan, X. Zhang, and Y. Wang, "Targeting JNK by a new curcumin analog to inhibit NF-kB-mediated expression of cell adhesion molecules attenuates renal macrophage infiltration and injury in diabetic mice," PLoS ONE, vol. 8, no. 11, Article ID e79084, 2013.

[49] W. L. Thompson and L. J. Van Eldik, "Inflammatory cytokines stimulate the chemokines CCL2/MCP-1 and CCL7/MCP-3 through $\mathrm{NF} \kappa \mathrm{B}$ and MAPK dependent pathways in rat astrocytes," Brain Research, vol. 1287, pp. 47-57, 2009.

[50] R. Safirstein, J. DiMari, J. Meavesi, and P. Price, "Mechanisms of renal repair and survival following acute injury," Seminars in Nephrology, vol. 18, no. 5, pp. 519-522, 1998.

[51] F. Xia, C. Wang, Y. Jin et al., "Luteolin protects HUVECs from TNF- $\alpha$-induced oxidative stress and inflammation via its effects on the Nox4/ROS-NF- $\kappa$ B and MAPK pathways," Journal of Atherosclerosis and Thrombosis, vol. 21, no. 8, pp. 768-783, 2014. 


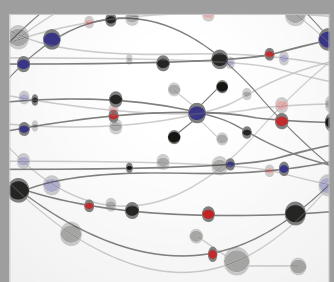

The Scientific World Journal
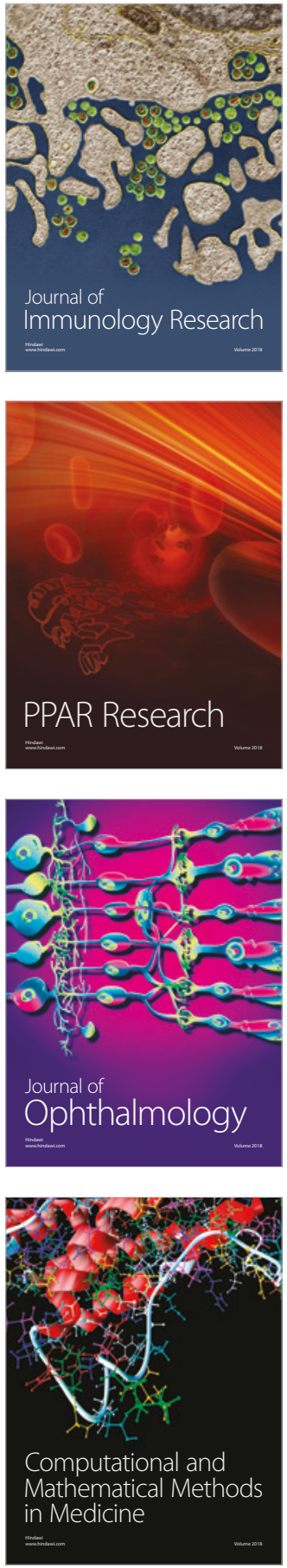

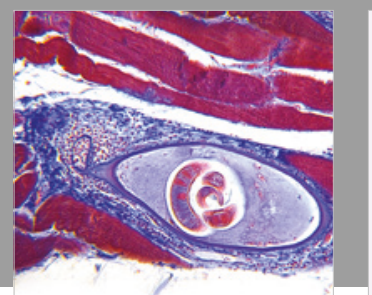

Gastroenterology Research and Practice

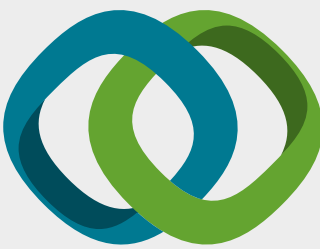

\section{Hindawi}

Submit your manuscripts at

www.hindawi.com
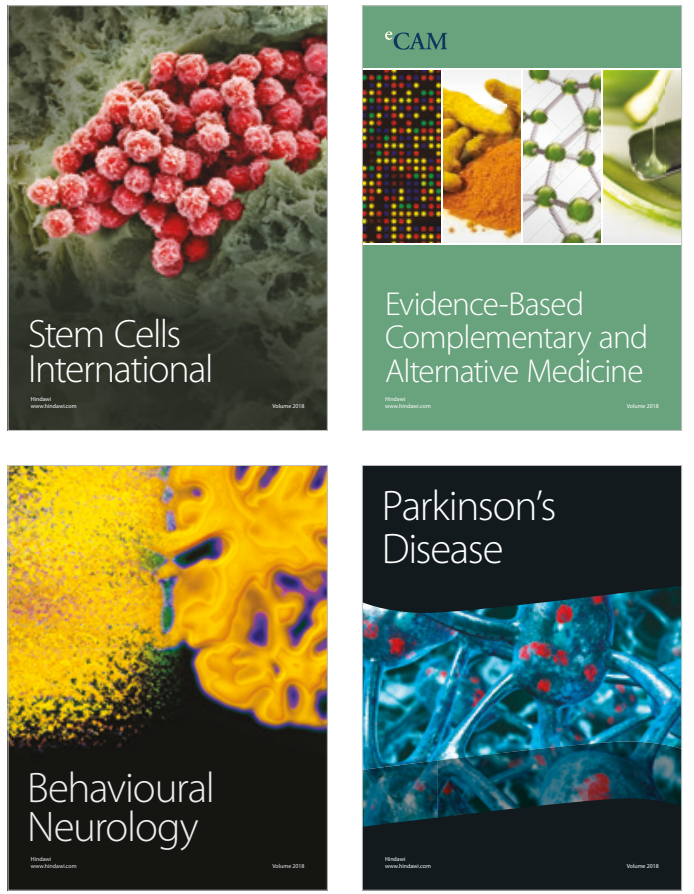

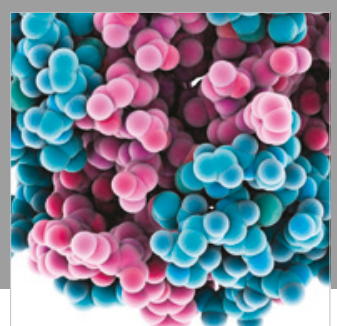

ournal of

Diabetes Research

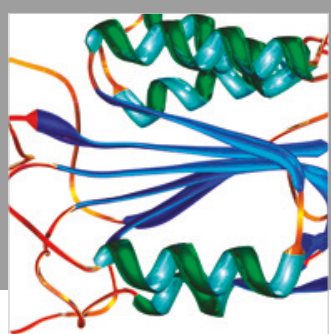

Disease Markers
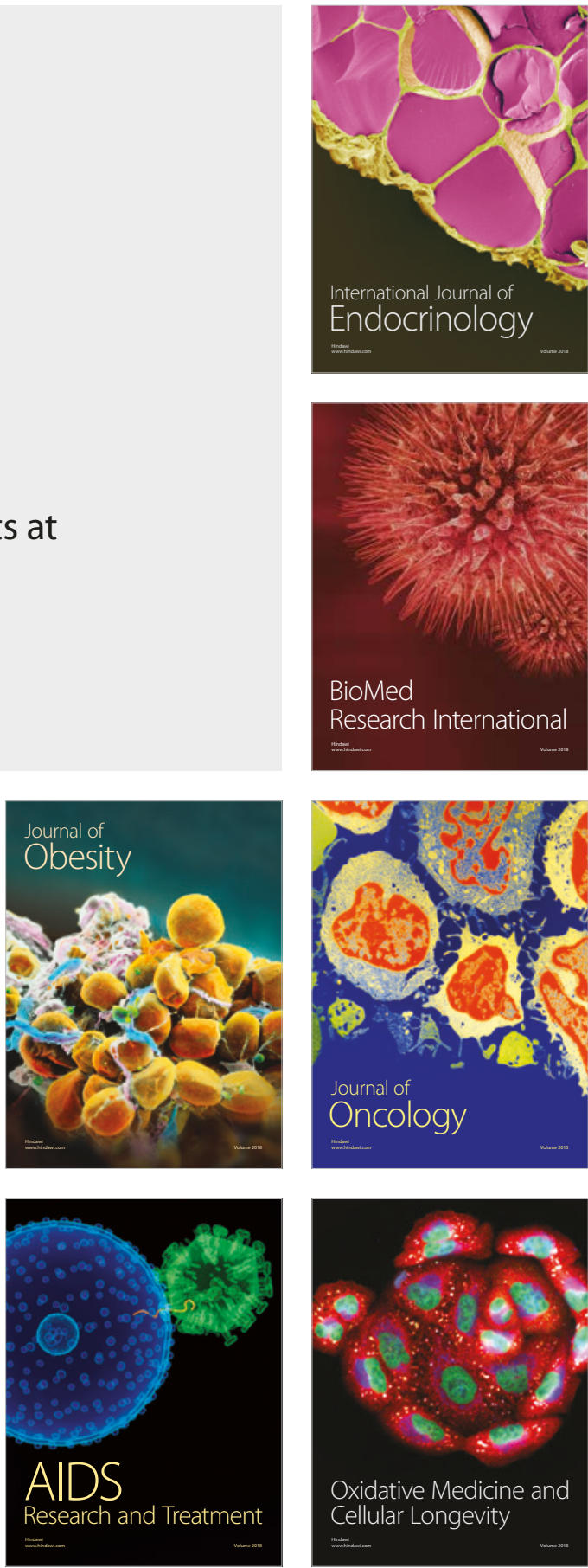